\title{
Linx
}

Revue des linguistes de l'université Paris X Nanterre

$41 \mid 1999$

L'hypothétique

\section{Genèse et interprétation des repères hypothétiques en milieu toncal - entre arbitraire et pertinence}

Jean-Marie Merle

\section{OpenEdition}

Journals

Édition électronique

URL : http://journals.openedition.org/linx/785

DOI : $10.4000 / \operatorname{linx} .785$

ISSN : 2118-9692

Éditeur

Presses universitaires de Paris Nanterre

Édition imprimée

Date de publication : 1 décembre 1999

Pagination : 61-74

ISBN : 0246-8743

ISSN : 0246-8743

Référence électronique

Jean-Marie Merle, «Genèse et interprétation des repères hypothétiques en milieu toncal - entre arbitraire et pertinence », Linx [En ligne], 41 | 1999, mis en ligne le 27 août 2012, consulté le 19 avril 2019. URL : http://journals.openedition.org/linx/785 ; DOI : 10.4000/linx.785

Ce document a été généré automatiquement le 19 avril 2019

Département de Sciences du langage, Université Paris Ouest 


\title{
Genèse et interprétation des repères hypothétiques en milieu toncal - entre arbitraire et pertinence
}

\author{
Jean-Marie Merle
}

1 Noncal (dérivé du latin nunc) et toncal (dérivé du latin tunc) sont des termes empruntés à J. Damourette et E. Pichon (1936/ 1970, tome $5: 174$ et suivantes). Le terme de noncal désigne le plan englobant les coordonnées-origine. Celui de toncal s'applique à tous les temps grammaticaux (imparfait, plus-que-parfait, conditionnel) situant un procès - et la relation nouée par ce procès - dans un plan (toncal) en rupture par rapport au plan des coordonnées-origine. Une note indiquera plus bas (note 9) la distinction que l'on fait entre rupture aoristique (directe) et rupture toncale, celle-ci correspondant à la structuration d'un plan toncal.

2 En milieu toncal- lorsque l'on rencontre les marqueurs des temps grammaticaux énumérés, ou marqueurs de toncalité, de repérage dans un plan toncal - un repère hypothétique n'est souvent identifiable en tant que tel, et son statut - «irréel ${ }^{1}$, potentiel, rhétorique - n'est intelligible ${ }^{2}$ - qu'en fonction de la conscience que l'on peut avoir du travail préalable dont il résulte et du préconstruit à partir duquel l'énonciateur lui confère le statut de repère hypothétique.

\section{Ambiguiité du conditionnel. Le statut de repère hypothétique}

\subsection{Rôle de l'interprétation}

(1a) Au sein du Canada, le Québec pèse plus que son poids démographique. Indépendant, ce serait un pays de second ordre, doté de la vingtième économie du monde, un îlot francophone aux rivages encore plus fortement battus par les vagues anglo-saxonnes. (Le Monde) 
(2a) Traduit en cinquante-sept langues, Simenon aurait vendu cinq cent cinquante millions d'exemplaires à travers le monde. (Le Monde) fonction du statut de la qualification apposée à gauche (Indépendant / Traduit en cinquantesept langues). Mais rien ne permet ici de discerner la pertinence et le statut de cette qualification - validée ou non validée - sinon l'accès au contexte élargi, et la conscience de la stratégie énonciative.

Dans le premier cas (Indépendant), la qualification est virtuelle, potentielle, dans un contexte où l'indépendance du Québec est le thème d'un débat, et ne peut résulter que d'un référendum qui n'a pas encore eu lieu. Il s'agit donc d'une qualification hypothétique, hypothème-repère sur lequel s'appuient l'écart toncal (la structuration d'un plan autre dans lequel l'issue du référendum serait une victoire du oui), l'orientation vers la validation et le caractère virtuel de l'apodose (ce serait un pays de second ordre).

5 Dans le second cas, la qualification est attestée (Traduit en cinquante-sept langues). Cette qualification a autant valeur d'explication que d'information. Si elle ne peut être comprise comme qualification hypothétique, à la différence de la précédente, c'est parce que le thème dominant n'est pas le nombre de langues dans lesquelles les œuvres de Simenon ont été traduites (?? Traduit en cinquante-six langues, si Simenon avait été traduit en cinquante-sept langues, il aurait vendu...), mais l'ampleur de leur diffusion. Il n'est donc pas nécessaire de savoir a priori que la qualification est attestée pour comprendre qu'elle l'est. Le conditionnel (aurait vendu) n'en demeure pas moins virtuel toncal ${ }^{3}$ : son emploi correspond ici à une prise de distance toncalisante et virtualisante justifiée par le caractère non-vérifié (non-vérifiable) du contenu propositionnel rapporté, à droite de la qualification (<Simenon-vendre cinq cent cinquante millions d'exemplaires>). On a ainsi affaire à un conditionnel journalistique, ou conditionnel de précaution.

6 L'interprétation du conditionnel dépend étroitement du statut de la qualification. La traduction en anglais ne sera possible qu'au prix de cette analyse préalable. A l'intérieur de l'apodose de l'exemple 1, la traduction de ce serait se fera à l'aide de would + BV :

(1b) Quebec carries out more clout in Canada than is warranted by its population's size. [ ${ }^{4}$ Independent ], it would be a second-class country with an economy ranking 20th in the world, a small Francophone island subject to even stronger pressures from the surrounding English-speakers. (The Guardian Weekly)

7 La traduction de l'exemple 2 lève d'emblée toute ambiguïté quant au caractère attesté de la qualification traduit en cinquante-sept langues :

(2b) Simenon has been translated into 57 languages. The total number of his books sold throughout the world is believed to be 550 million. (The Guardian Weekly)

8 La traduction du conditionnel «aurait vendu» reflète ensuite les résultats de l'analyse. L'absence d'orientation vers la validation interdit l'emploi de would + BV : on n'a affaire ni à l'expression d'un probable, ni à une prédiction, ni à l'expression du vouloir. L'énonciateur, on l'a vu, toncalise et virtualise un contenu propositionnel dont il ne se porte pas garant car il n'y a pas eu accès de première main (conditionnel de précaution, encore appelé conditionnel de reprise). Le traducteur opte ici pour une modulation diathétique ( $S$ is believed to $P$ ), à la faveur de laquelle il introduit sans mentionner sa source, la trace d'un repérage intermédiaire. 


\subsection{Ambiguïté du conditionnel. Rôle de la pertinence}

9 Ainsi encore la phrase-type suivante :

(3) S'il faisait beau, j'irais me promener.

est susceptible de deux interprétations différentes :

- système hypothétique «irréel » (contrefactuel : hypothème forclos) : il ne fait pas beau ;

- système hypothétique potentiel (hypothème non forclos) : il n'est pas exclu qu'il fasse beau.

11 Cette phrase ne devient énoncé et n'acquiert d'intelligibilité qu'en fonction de la pertinence des choix notionnels (faire beau / aller se promener) - de leur lien avec le préconstruit situationnel ou contextuel (Il ne fait pas beau / Il n'est pas exclu qu'il fasse beau) et de leur lien avec le thème ambiant (Le temps qu'il fait ou qu'il fera // Sortir / rester à la maison).

\subsection{Le repère hypothétique}

Soit l'énoncé :

(4) [...] \{ si j'étais à ta place \}, je ferais réparer cette marche au plus vite. (Les Bijoux de la Castafiore, p. 17)

13 L'énonciateur construit son énoncé sur une hypothèse, littéralement: proposition posée en dessous, qui tient lieu de fondement, de base à un raisonnement, à un discours. La première proposition, si $p$ (si j'étais à ta place), est traditionnellement appelée protase (proposition qui tient lieu de prémisse majeure d'un argument, première partie d'une période, proposition à développer). Sur le soubassement fourni par la protase s'appuie l'apodose $q$ (je ferais réparer cette marche au plus vite), qui en est le développement, et dont le contenu propositionnel ne vaut que repéré par rapport au repère hypothétique si $p$.

M. Maillard (1985 : 98) donne du terme thétique la définition suivante :

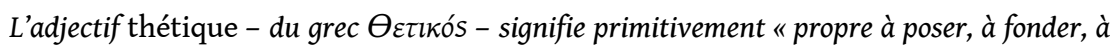
établir ". Une proposition thétique est donc une proposition dont le rôle est de poser un thème nouveau [...].

Ce terme s'appliquerait à toute protase de manière générique dans le sens où l'énonciateur pose un thème comme soubassement (hypo-) repère (circonstant hypothétique) de son apodose ${ }^{5}$, mais selon plusieurs schémas d'application.

\subsection{Genèse des repères hypothétiques. Trois cas de figure}

Schéma de l'« irréel » (ou contrefactuel : «Altérons $\left.p^{6} »\right)$ : altération (de $p$ à si p' ou de p' à si p)

(4) \{Si j'étais à ta place\}, je ferais réparer cette marche au plus vite. (Les Bijoux de la Castafiore, p. 17).

(5) \{ Si je n'avais pas eu l'attention attirée par ces mouettes \}, nous étions aplatis... (Le Crabe aux pinces d'or, p. 9)

(6) [...],\{ si ces grenades avaient été chargées au lieu de n'être qu'amorcées \}, nous ne serions plus de ce monde... (Les Cigares du Pharaon, p. 22)

L'hypothèse de chacun de ces trois énoncés ne se construit pas ex nihilo. Elle résulte dans chacun des cas d'une opération d'altération d'un préconstruit. 

locuteur a été attirée par des mouettes $(p) ;(6)$ les grenades étaient amorcées $(p)$, mais non chargées. Ce préconstruit, situationnel dans les trois cas, fait partie des connaissances partagées par le locuteur et son allocutaire. l'hypothèse si $p$ par altération du préconstruit $\left.p^{\prime}\right)$; (5) Si je n'avais pas eu l'attention attirée par ces mouettes (genèse de l'hypothèse si p' par altération du préconstruit $p$ ) ; (6) Si ces grenades avaient été chargées (genèse de l'hypothèse si p'-grenades chargées - par altération du préconstruit $p$ - grenades seulement amorcées). Dans les deux premiers cas l'opération d'altération porte sur la polarité : (4) de non pà si p / (5) de pà si non p. Dans le troisième cas (6), l'opération d'altération est matérialisée ( au lieu de), et la substitution des prédicats apparaît en surface, le préconstruit est à la fois situationnel et contextuel : de $p$ (grenades amorcées) à si p' (grenades chargées), autre que $p$. hypothétique. Elle est donc doublement repérée :

- d'une part, par rapport au repère hypothétique ;

- d'autre part, par rapport à un préconstruit: (4) Personne ne s'occupe de faire réparer cette marche ; (5) Nous n'avons pas été aplatis ; (6) Nous sommes encore de ce monde.

La pertinence des choix notionnels constitutifs du contenu propositionnel de l'apodose s'appuie sur ce double repérage. L'apodose a été décrite comme un développement construit sur le soubassement de la protase. Ce développement relève de la stratégie énonciative et contribue en retour à la pertinence de l'altération.

On présentera ici la pertinence comme le complémentaire et le contrepoids de l'arbitraire. Les gloses suivantes ont pour objet de tenter d'illustrer la place de l'arbitraire et celle de la pertinence :

(4) Je me substitue à vous (à titre d'hypothèse : arbitraire) et ce faisant je me mêle de ce qui ne me regarde pas (risque d'irrecevabilité de l'arbitraire), mais vous avez une marche à faire réparer et dangereuse dans l'état où elle est (pertinence établie par le lien avec le préconstruit situationnel) et vous ne la faites pas réparer (préconstruit), alors en me substituant à vous, je vous dis ce que je ferais à votre place et je vous donne un bon conseil.

(5) A titre d'hypothèse (arbitraire), je pose comme n'ayant pas été le cas ce qui a été le cas (attention attirée par des mouettes). A la faveur de cette altération, je reformule (j'altère) les faits (nous étions aplatis). Préconstruit situationnel: une caisse vient de s'écraser à côté du locuteur (lien avec le préconstruit garant de la pertinence de l'énoncé). Il s'en est fallu de peu.

(6) A titre d'hypothèse (arbitraire), je pose comme validé dans sa relation avec le sujet un prédicat autre (grenades chargées) que celui qui entre dans la relation validée formulant le préconstruit (grenades amorcées). Mais il est bien connu qu'une grenade peut être chargée (pertinence notionnelle de l'altération) aussi bien qu'amorcée. Nous avons eu de la chance. 


\section{Altération et rupture toncale}

\subsection{Le plan toncal} comme un «irréel » (contrefactuel).

Schéma du potentiel ( $($ Imaginons $p »)$ : de $\varnothing$ (ou de p,p') à si $p$ tel que si $p\left[p,\left(p^{\prime}\right)\right]$ : altérité

(7) \{ Si l'on trace un triangle équilatéral \}... (Soit un triangle équilatéral...)

(8) S'il fait beau dimanche, je vais (j'irai) me promener.

(3') S'il faisait beau dimanche, j'irais me promener. potentiel paraît à première vue plus authentiquement thétique, dans le sens où il semble évoqué ex nihilo. La représentation donnée ci-dessus - de Ø ou de $p, p^{\prime}$ à si $p\left[p,\left(p^{\prime}\right)\right]$ - hésite entre :

- l'évocation ex nihilo et ex abrupto (genèse à partir de Ø) dans un cadre où l'absence de lien avec le thème ambiant ${ }^{8}$ ôte toute pertinence au thème inauguré, l'exposant au risque d'irrecevabilité, d'une part ;

- et, d'autre part, l'évocation d'un possible (genèse à partir de $p, p^{\prime}$ ) dont la pertinence notionnelle est garantie par son lien avec les préconstruits thématiques, situationnels ou contextuels.

L'ensemble peut se lire : «la notion $p, p^{\prime}$ étant pertinente, $p$ n'étant pas forclos est pris comme repère hypothétique - si $p$, tel que $\left[p,\left(p^{\prime}\right)\right]$, c'est-à-dire tel que $p$ est envisagé comme repère hypothétique sans pour autant que $p$ ' soit exclu (altérité caractéristique du possible et du virtuel) ».

La pertinence du triangle équilatéral évoqué en 7 découlera du lien avec la situation construite - un cours de géométrie, d'architecture, de dessin... seront respectivement appelées potentiel noncal et potentiel toncal. 
Dans les deux cas, a-t-on remarqué, elles reçoivent leur pertinence de leur lien avec un thème ambiant (beau temps / mauvais temps // Que faire dimanche ? sortir / rester), et c'est à cette condition seulement que ces deux phrases pourront devenir énoncés.

Mais la pertinence du repère hypothétique S'il fait beau est directe, noncale : beau temps, je sors, (pluie, je reste).

Tandis que la pertinence du repère hypothétique S'il faisait beau est indirecte, toncale. L'emploi de l'imparfait (toncal) implique en effet la construction d'un plan toncal : a priori je reste à la maison, mais [écart toncal] s'il faisait beau, j'irais me promener / S'il faisait beau [j'ai des doutes mais je veux bien formuler cette hypothèse, au prix d'un écart toncal]... / [De telles considérations n'ont pas de pertinence directe - on verra bien dimanche - mais après tout : écart toncal ] s'il faisait beau...

\subsection{Schéma de la reprise}

de $p$ à si $p$ (hypothème rhétorique : «Admettons $p$ »)

(9) - Je suis sûr que ce sont les restes des pirates tués par l'explosion de la LICORNE!

- Sûrement pas, capitaine...

... car, $\{$ dans ce cas $\}$, nous les aurions découverts près du rivage. (Le Trésor de Rackham le Rouge, p. 27)

(10a) Tchang n'est pas mort. (préconstruit, p. 5)

(10b) \{ Si votre ami Tchang n'était pas mort \}, l'expédition de secours l'aurait retrouvé. (Tintin au Tibet, p. 6)

Dans ces deux exemples, la protase - (9) dans ce cas / (10) Si votre ami Tchang n'était pas mort - est reprise d'un contenu propositionnel fourni en amont et qui garantit la pertinence de l'hypothème : (9) ce sont les restes des pirates tués par l'explosion de la Licorne / (10) Tchang n'est pas mort.

Dans les deux cas, le locuteur concède ${ }^{9}$ au co-locuteur et ce contenu propositionnel et la polarité qui lui est associée : admettons que tel soit le cas. La toncalité de l'apodose reflète ( virtuel toncal antérieur) une altération rétrospective matérialisée par la représentation explicite d'un virtuel dans un plan toncal ((9) : nous les aurions découverts / (10) : l'expédition de secours l'aurait retrouvé), altération respectivement du préconstruit : (9) nous ne les avons pas découverts près du rivage / (10) l'expédition de secours ne l'a pas retrouvé).

41 La toncalisation du contenu propositionnel repris est explicite - (10a) Tchang n'est pas mort / (10b) Si votre ami Tchang n'était pas mort - ou implicite - (9) Dans ce cas = Si c'étaient les restes des pirates... : elle annonce la stratégie énonciative et amorce, en le situant dans un plan toncal, le travail de mise en doute de ce contenu propositionnel.

4 Dans ces deux exemples, la reprise est rhétorique dans la mesure où le système hypothétique construit sur cette reprise constitue la première prémisse d'un syllogisme implicite. Prémisse 1 explicite : (9) Dans ce cas (en admettant ce que vous avancez: si p), alors $q$ : nous les aurions découverts près du rivage / (10) Si [effectivement] Tchang n'était pas mort: si $p$, alors $q$ : l'expédition de secours l'aurait retrouvé. Prémisse 2, préconstruit: (9) Nous ne les avons pas découverts près du rivage (q') / (10) L'expédition de secours ne l'a pas retrouvé (q'). Conclusion: (9) Sûrement pas - explicite - (Ce ne sont pas les restes des pirates tués par l'explosion de la Licorne) / (10) Tchang ne peut pas être vivant - implicite.

La construction ternaire du syllogisme s'appuie sur le lien de solidarité (d'implication) entre protase et apodose. 


\subsection{Remarque sur le rôle de la pertinence dans l'opposition concessive}

(11) [...] \{ même si votre ami avait survécu à l'accident \}, il serait mort depuis, de

faim, de froid, d'épuisement. (Tintin au Tibet, p. 10)

La stratégie énonciative consiste à opposer deux contenus propositionnels :

- d'une part, l'hypothème-repère, concédant au co-locuteur ce que celui-ci tient pour validé et pertinent ;

- d'autre part, un contenu propositionnel repéré, le propos du locuteur, qui estompe et virtualise la pertinence du contenu propositionnel concédé.

La concession du premier contenu propositionnel (même si $p$, ...) pourrait se gloser : en admettant ce que vous croyez, de toute façon, ..., admettons ce que vous croyez, mais en toute hypothèse, ..., allons jusqu'à admettre ce que vous croyez, mais c'est égal, ..., admettons, mais cela ne change rien, .... Que $p$ (qu'il ait survécu à l'accident) soit validé importe peu - il importe peu que ce soit $p$ ou $p^{\prime}$ - puisque $q$ est quasi-certain : de toute façon - qu'il ait survécu ou non ( $\left.p, p^{\prime}\right)$ - il serait mort depuis (q).

L'opposition concessive se construit sur la hiérarchisation de la pertinence des deux contenus propositionnels, la pertinence de $q$ venant ici supplanter la pertinence de $p$.

\subsection{Arbitraire absolu et irrecevabilité de la stratégie énonciative}

(12) Le professeur : Vous avez... vous avez... vous avez...

L'élève : Dix doigts !...

Le professeur : Si vous voulez. Parfait. Bon. Vous avez donc dix doigts.

L'élève : Oui, Monsieur.

Le professeur : Combien en auriez-vous, si vous en aviez cinq?

L'élève : Dix, Monsieur. (E. Ionesco, La leçon, p. 110)

L'énoncé Combien en auriez-vous, si vous en aviez cinq? est conçu pour mettre a nu l'arbitraire de l'hypothèse, arbitraire thétique qui constitue l'un des ressorts essentiels de l'absurde, et qui favorise son irrecevabilité.

L'absence de pertinence de l'hypothèse tient, en premier lieu, à l'absence de pertinence de la question (Combien en auriez-vous... ?), privée de fonction dans l'élaboration de l'énoncé puisque la réponse est formulée dans le segment-repère (si vous en aviez tant).

Pour la même raison, l'apodose (vous en auriez tant) perd toute pertinence puisque son contenu référentiel virtuel est identique à celui de la protase (si vous en aviez tant), et qu'elle est ainsi privée de fonction à l'intérieur du système hypothétique.

La stratégie énonciative ne tient plus qu'à un fil mis à nu: la genèse arbitraire, par altération du préconstruit $p$ (Vous avez donc dix doigts) d'un hypothème dénué de pertinence, si p' (... si vous en aviez cinq) et n'ouvrant que sur lui-même - c'est-à-dire sur une impasse - ne signifiant que l'arbitraire.

51 On se rappellera l'axiome Avec des «si ».... , qui a précisément pour fonction, en situation, d'exprimer la conscience de l'arbitraire (hypo-) thétique (et énonciatif), et l'irrecevabilité de cet arbitraire. 


\section{Conclusion} travail énonciatif dans lequel arbitraire et pertinence jouent un rôle complémentaire, reflet de l'intention de signifier et de la stratégie énonciative. La conscience de la genèse des repères hypothétiques, de leur pertinence notionnelle, situationnelle, contextuelle ou stratégique, est indispensable pour que ces repères soient identifiables, interprétables, intelligibles ou même recevables.

\section{BIBLIOGRAPHIE}

DAMOURETTE, J. et PICHON, E.. (1936), Des mots à la pensée. Essai de grammaire de la langue française, tome V (1911-1936), Paris, Editions d'Artrey, réimp. 1970.

DELMAS, C. (1994), « Remarques sur le passif impersonnel de it à there ", L'information grammaticale, $\mathrm{n}^{\circ} 62$, juin.

LE GOFFIC, P. (1986), «Que l'imparfait n'est pas un temps du passé », Points de vue sur l'imparfait, Centre de Publications de l'Université de Caen, pp. 55-69.

LE GOFFIC, P. (1993), Grammaire de la phrase française, Paris, Hachette. 
MAILLARD, M. (1985), « L'impersonnel français de il àça », Autour de l'impersonnel, Grenoble, ELLUG, pp. 63-118.

MAINGUENEAU, D. (1991b), L'Énonciation en linguistique française, Paris, Hachette.

LARREYA, P. (1984), Le possible et le nécessaire : modalités et auxiliaires modaux en anglais britannique, Paris, Nathan Recherche.

MERLE, J.-M. (1998), « De la projection hypothétique à l'atténuation, conditionnel français vs WOULD + BV », Linguistique contrastive et traduction, tome 4, Paris, Ophrys, pp. 115-185.

TOURATIER, Ch. (1996), Le système verbal français, Paris, Armand Colin.

TRÉVISE, A. (1994), Le prétérit anglais, Paris, Nathan.

de VOGUË, S. (1986), « La conjonction si et la question de l'homonymie », BULAG 13, Université de Besançon, pp. 105-189.

WAGNER, R.L. et PINCHON, J. (1991), Grammaire du français classique et moderne, Paris, Hachette.

WILMET, M. (1997), Grammaire critique du français, Paris, Duculot, Hachette Supérieur.

\section{NOTES}

1. Le terme d'«irréel» a été conservé ici parce qu'il appartient aux conventions et bien qu'il soit particulièrement trompeur pour la simple raison qu'il s'oppose à "réel », que langue et langage ont un champ d'application qui dépasse nécessairement la simple description du réel et que toutes les opérations énonciatives sont mises à contribution pour structurer de l'irréel.

C'est pour cette même raison que le terme de conditionnel a été conservé, bien que ce terme soit également trompeur: il est en effet impossible de retrouver une condition derrière chaque emploi du conditionnel. Ex. : Henri IV, qui serait assassiné en 1610, eut néanmoins le temps d'assainir les finances $d u$ pays. (conditionnel archétypique de prédestination). On peut se demander quelle condition remplacer. ?? si son destin s'accomplissait... ? La recherche d'une condition n'a aucune chance d'aboutir... Il en est de même dans les énoncés contenant un conditionnel journalistique, ludique, conjectural, hypocoristique, ainsi que dans les prédictions rapportées. On le nomme donc conditionnel mais, parallèlement, on le décrira comme virtuel toncal (cf. ci-dessous note 4).

2. Certains cas sont sans ambiguïté. Ainsi les protases canoniques en $s i+$ plus-que-parfait (ou toncal antérieur, cf. exemples 5 et 6), dont le contenu propositionnel est nécessairement contrefactuel («irréel »).

3. Virtuel toncal: résultant de la fusion [infinitif V.lexical+avoir imparfait], le conditionnelcorrespond à la localisation d'un virtuel (morphème-R-de l'infinitif) dans un plan toncal - plan autre, en rupture par rapport à celui des coordonnées-origine - (désinence -ais de l'imparfait).

Remarque : il est difficile d'adopter l'idée que le conditionnel $=*$ [futur + imparfait $]$ étant donné que le futur résulte d'une formation analogue : futur = [infinitif V. lexical + avoir présent $]$. Ce qui reviendrait à dire $:$ conditionnel $=*$ [infinitif $\mathrm{V}$. lexical + avoir présent + imparfait $]$.

4. Les accolades sont employées pour signaler l'élément hypothétique.

5. A partir de cette définition, on établira une distinction entre hypothèse et conjecture. La conjecture, en tant qu'expression d'un problème de connaissance, entre dans le paradigme des modalités épistémiques. Elle peut être conclusive (Tiens, tiens, Paul aurait donc oublié de fermer à clef ) ou non conclusive (Ah çà! où donc est-il passé? Serait-il dans l'arbre? [Les cigares du Pharaon]). L'hypothèse, en revanche, donne lieu à un développement.

Cette distinction ne signifie nullement que la conjecture ne puisse devenir hypothèse, mais à 
condition qu'elle devienne le soubassement d'un développement. Ainsi \{ Ils auraient voulu nous flanquer dans le lac \} qu'ils ne s'y seraient pas pris autrement (L'affaire Tournesol, p. 21) : la première proposition est conjecturale, interprétation (explicitement virtuelle toncale)d'une situation à partir d'indices (Tiens, tiens... tout porte à croire qu'on a voulu nous flanquer dans le lac), tandis que la seconde, liée à la première et par que et par le parallélisme (-rait / -rait) qui solidarisent les deux contenus propositionnels, en est le développement, confirmation de l'adéquation (ne... pas... autrement) de la conjecture aux indices situationnels. L'ensemble peut se gloser à l'aide d'un système hypothétique canonique : S'ils avaient voulu nous flanquer dans le lac, ils ne s'y seraient pas pris autrement.

6. Par convention $p$ symbolise une occurrence (simple ou complexe : il peut s'agir d'un contenu propositionnel) validée et s'oppose à $p^{\prime}$, qui symbolise une occurrence non-validée. (p,p') représente les deux valeurs couvrant l'étendue du domaine notionnel, l'hésitation ou la neutralité entre validation et non-validation caractéristique du virtuel ou du possible.

7. La rupture toncale correspond à la structuration d'un plan autre, et s'oppose en cela à la rupture aoristique.

Exemple 1. Il trouva qu'elle était belle. La relation nouée autour du procès trouva est en rupture directe, aoristique, par rapport aux coordonnées-origine (il la trouva belle à ce moment-là, au moment où il la vit) : la perception (il trouva) entre dans l'enchaînement aoristique / le contenu propositionnel de la complétive (qu'elle était belle) est repéré par rapport au procès exprimant la perception : repérage (toncal) dans un plan (toncal) construit à partir du repère (en rupture par rapport aux coordonnées-origine) il trouva. Ce type de repérage entraîne systématiquement l'emploi d'un toncal (cf. *Il trouva qu'elle fut belle). C'est par le biais de ce repérage que l'imparfait est apte à exprimer un procès dont le référent coïncide avec le moment d'énonciation ou lui est postérieur. On pourra énoncer Dire qu'il se mariait aujourd'hui / la semaine prochaine (« il fut un temps où il était prévu qu'il se mariait aujourd'hui ») : la construction de deux plans (noncal et toncal) différents permet leur intersection. En revanche, on ne peut énoncer *Dire qu'il se maria aujourd'hui / la semaine prochaine (une dépendance de ce type, toncale, étant exclue par définition $\mathrm{du}$ fonctionnement aoristique, qui correspond à une rupture directe).

Exemple 2 : Elle crut qu'il viendrait. Même type de repérage de il viendrait dans un plan toncal. Mais on a affaire ici à un virtuel toncal (conditionnel) (cf. *J'ai cru qu'il viendra).

Exemple 3 : Si j'étais vous, je la ferais réparer. Construction ipso facto d'un plan toncal en raison de l'altération. (cf. *Si je fus vous / Si je suis vous / Si j'étais vous, je la *ferai réparer).

Exemple 4: Alors on avait un gros chagrin. L'emploi de l'imparfait (ici hypocoristique) suffit à construire un plan toncal, sans passer par la dépendance d'un repérage autre explicite. (cf. Alors on *eut un gros chagrin / Alors, on a un gros chagrin : plan noncal, pertinence directe : ici, seul l'emploi de on est hypocoristique).

Exemple 5 : Je serais le roi, tu serais le capitaine des gardes... Le conditionnel (virtuel toncal) suffit à construire un plan toncal. Ici, conditionnel ludique, qui structure un univers virtuel.

8. Si l'on imagine un boucher déclarant à une cliente qui vient d'entrer dans sa boutique : Soit un triangle équilatéral..., ou encore un mathématicien travaillant devant un auditoire de confrères sur un problème de géométrie analytique et leur annonçant: J'aime le chocolat, on a affaire à deux énoncés thétiques dont l'arbitraire n'est compensé par aucune pertinence.

Le plus souvent, l'inauguration d'un thème s'accompagne de précautions oratoires destinées à atténuer en l'annonçant les effets de l'arbitraire thétique, et à compenser l'absence de pertinence d'un énoncé (Ah oui, au fait, il y a Jean qui a appelé ce matin), ou d'opérations de repérage destinées à fournir au thème inauguré un cadre recevable (Dans le présent ouvrage, le lecteur, même débutant, trouvera...).

9. Le terme de concession n'est pas utilisé ici dans le sens d'opposition concessive.

10. ... on mettrait Paris en bouteille. 


\section{AUTEUR}

\section{JEAN-MARIE MERLE}

Université de Provence

Centre des lettres et sciences humaines

Université de Provence

29, avenue Robert Schuman

13621 - Aix-en-Provence Cedex 1 